Burmeister, Edwin, and A. Rodney Dobell

"Guidance and Optimal Control of Free-Market Economies: A New Interpretation." IEEE Transactions on Systems, Man and Cybernetics SMC-2.1(1972): 9-15.

Reprinted with permission from The Institute of Electrical and Electronics Engineers, Inc. 


\title{
Guidance and Optimal Control of Free-Market Economies: A New Interpretation
}

\author{
EDWIN BURMEISTER AND A. RODNEY DOBELL
}

\begin{abstract}
The biological and social sciences offer many examples of complex systems which were not consciously designed or engineered but which have evolved over long periods from more primitive forms. Built-in mechanisms for control or guidance of/ such systems may be quite elaborate but somewhat imperfect and indirect. Two primary propositions are discussed. The first is that the evolution of the complex markets basic in western economies has realized an institutional structure through which decentralized guidance of the economy can be implemented. The second is that with such market structures it may be possible to design economic policies which realize sufficient control of the economic system without direct intervention in the optimizing decisions of individual elements in the system. The paper is primarily tutorial and surveys the relevant technical literature on models of economic growth. However, some new results and a new interpretation of known results are presented.
\end{abstract}

\section{INTRODUCTION}

$\mathrm{F}$ OR SOME PURPOSES of analysis-in particular, for our present expository purposes - an economy may be viewed as a system in which the inputs are stocks of productive assets or resources (a complete inventory of the machines, manpower, and material stocks available for production), and the outputs are flows of goods and services for final consumption or for addition to the stocks of productive resources available for later use in creation of still further goods. To illustrate our discussion in concrete terms, we first consider a particularly simple example of such a system-one in which there is only a single produced asset, called capital equipment, the stock of which at time $t$ is denoted by $K(t)$. The output of this system is divided into two flows: a flow of goods intended for final consumption and a flow of investment goods to be added to the stock of capital equipment. Denoting these two flows by $C(t)$ and $I(t)$, respectively. we recognize that physical considerations impose the constraints $I \geq 0, C \geq 0$. We also assume that technological relations (the "plant" for the economic system) may be summarized in the equations

$$
\begin{aligned}
C+I & =F(K) \\
\dot{K} & =I-\delta K
\end{aligned}
$$

which describe the stream of output $F(K)$ attainable from the services of a given stock $K$ of capital equipment and prescribe that this output flow be allocated either to consumption $C$ or to gross investment $I$. In turn, investment

Manuscript received February 15, 1971; revised August 13, 1971. This work was supported by the National Science Foundation under Research Grant GS-3249 and by the Canada Council through a Grant from the lzaak Walton Killam Memorial Fund. This paper was presented in part at the 1969 IEEE Systems Science and Cybernetics Conference, Philadelphia, Pa., October 22-24.

E. Burmeister is with the Department of Economics, Duke University, Durham, N.C. 27706, on leave from the Department of Economics, University of Pennsylvania, Philadelphia, Pa. 19104.

A. R. Dobell is with the Department of Economics, University of Toronto, Toronto, Ont., Canada. expenditure leads, after deduction of provision for replacement of equipment at a rate $\delta K$, to further accumulation of asset stocks as shown in (2). Finally, we suppose that economic theory predicts an aggregate saving function $s(t)$ describing the composition of output at each moment so that one has

$$
I(t)=s(t) F(K(t)) .
$$

(The nonnegativity constraints cited earlier imply that one has the restriction $0 \leq s \leq 1$, for all $t$.)

It is clear that this model, though crude, does describe a complete system, and it could be used to study some simple features of a process of capital accumulation and economic growth. Equations (1)-(3) thus constitute, for given functions $F$ and $s$ and given initial condition $K(0)=K_{0}$, a simple differential equation describing an economic system. From a given starting point the whole future of this model economy is determined. Descriptive theories of economic growth study the consequences of various arbitrary rules for obtaining the function $s$ and thus determining the composition of output; stability properties of the solutions to the resulting differential equations have been of particular interest [10], [19]. Here, however, we wish to consider how such saving rules might be derived from performance criteria reflecting considerations of social welfare.

Imagine, for example, that a new central government is elected for a term of $T$ years on a platform which promises "to determine economic policy so as to realize the maximum attainable value of a social welfare criterion

$$
J=\int_{0}^{T} U(C(t)) \exp (-\gamma t) d t
$$

where $U$ is a specified smooth concave positive function and $\gamma$ a fixed nonnegative number, subject to the technological constraints cited in the foregoing, given an initial capital stock and the pledge that the terminal capital stock shall equal a prescribed value $K_{T}$." Let us assume, since the voters approved the proposal, that this criterion accurately reflects the desires of the community. (The constant $\gamma$ would then be called, in economic jargon, the social rate of time preference for the community. The weighting function $\exp (-\gamma t)$ would thus assign less significance to consumption more distant in time, and the "utility function" $U$ would assign less weight to an increase in consumption the greater the size of the consumption flow already attained. Both weighting procedures are considered to reflect features of social preference, and the criterion function (4) is quite common in the literature on economic growth.) Then implementation of the proposal requires solution of a standard problem in the theory of optimal control. 


\section{Necessary and Sufficient Conditions for Optimal Control of the Illustrative Economic System}

The problem being so straightforward, we need only sketch the conditions characterizing an optimal policy. Following the conventional approach, we introduce an auxiliary variable $\psi$ and form a Hamiltonian given by the following equation:

$$
H=U((1-s) F(K)) \exp (-\gamma t)+\psi[s F(K)-\delta K] \text {. }
$$

As is well known, the maximum principle asserts that a necessary condition for an admissible policy $s(t)$ to be optimal is that there exist a continuous function $\psi(t)$ satisfying

$$
\begin{aligned}
\dot{\psi}=-\frac{\partial H}{\partial K}=-U^{\prime}((1-s) F(K)) & \exp (-\gamma t)(1-s) F^{\prime}(K) \\
& -\psi\left[s F^{\prime}(K)-\delta\right]
\end{aligned}
$$

and such that for fixed $K$ and $\psi, H$ is a maximum with respect to $s$ satisfying $0 \leq s \leq 1$. Because the functions $F$ and $U$ are both assumed concave, it can be proved that these necessary conditions are also sufficient conditions that a policy $s$ be optimal (see Mangasarian [12]).

Introducing a rescaled variable

$$
p=\exp (\gamma t) \psi
$$

and for convenience observing that $I(t)=s(t) F(K(t))$, we may rewrite the preceding necessary conditions as

$$
\begin{aligned}
\dot{K} & =I-\delta K \\
K(0) & =K_{0} \\
\dot{p} & =-U^{\prime}(F(K)-I) F^{\prime}(K)+(\gamma+\delta) p \\
p(T) & =p_{T}
\end{aligned}
$$

where $p_{T}$ is to be chosen to ensure that $K(T)=K_{T}$, and

$$
\begin{gathered}
U^{\prime}(F(K)-I) \geq p \\
\left(U^{\prime}-p\right) I=0 \\
I \geq 0, \quad K \geq 0, \quad p \geq 0 .
\end{gathered}
$$

We wish to analyze the nature of solutions to this system. First, let us consider the possibility of an equilibrium state $\dot{K}=0, \dot{p}=0$. Such an equilitrium would require the following equations:

$$
\begin{aligned}
I & =\delta K>0, \quad \text { if } K>0 \\
p & =\frac{U^{\prime}(F(K)-\delta K) F^{\prime}(K)}{(\gamma+\delta)} \\
& =\frac{p F^{\prime}(K)}{\gamma+\delta}, \quad \text { if } K>0 .
\end{aligned}
$$

Thus, if there is to be any positive equilibrium solution, it can be only at a value $K^{*}$ such that

$$
F^{\prime}\left(K^{*}\right)-\delta=\gamma
$$

that is, at a capital stock such that the rate of return (net of depreciation) is equal to the imposed rate of time preference $\gamma$. At this capital stock a constant equilibrium price given by the equation

$$
p^{*}=U^{\prime}\left(F\left(K^{*}\right)-\delta K^{*}\right)
$$

\section{is determined. ${ }^{\prime}$}

Considering construction of a phase diagram for the system and turning to trajectories with changing prices and stocks, it is helpful to determine the locus of points on which one or another of the variables is momentarily constant. For the case in which there is equality between what we may call the demand price $p$ and the supply price $U^{\prime}(C)$ of the investment good, we may write the price equation either as $\dot{p} / p=-\left[F^{\prime}(K)-(\gamma+\delta)\right]$ or as $\dot{U}^{\prime} / U^{\prime}=-\left[F^{\prime}(K)-(\gamma+\delta)\right]$, the latter equation eliminating all reference to the price $p$. From the former equation we conclude that $\dot{p}$ is zero only on the locus of points $K=K^{*}$ in the $(K, p)$ plane.

Inverting the side condition $U^{\prime}(F(K)-I)=p$, we obtain $I=-U^{\prime-1}(p)+F(K)$, and observing that the condition $\dot{K}=0$ requires $I=\delta K$, we may conclude that $\dot{K}$ is zero only on the locus of points satisfying the following equation:

$$
F(K)-\delta K=U^{\prime-1}(p) .
$$

With this information, it is possible to construct a phase diagram for the system, and thus to illustrate what the value of $p_{T}$ must be to ensure satisfaction of the pledge that the terminal capital satisfy $K(T)=K_{T}$. Hence we may characterize the accumulation path to be followed, observing in particular that the point $\left(K^{*}, p^{*}\right)$ constitutes a saddle point equilibrium for the system. Since the analysis is straightforward, we omit construction of such a phase diagram here.

It might happen that capital is initially so plentiful that all output can be consumed. In this case we must deal explicitly with the boundary case $I=0$. The system equations under these circumstances become

$$
\begin{aligned}
\dot{K} & =-\delta K \\
\dot{p} & =-U^{\prime}(F(K)) F^{\prime}(K)+(\gamma+\delta) p
\end{aligned}
$$

with appropriate boundary conditions. One possible sequence of events, then, is that initially capital is so plentiful that all output may be consumed. In this situation capital decumulates, and the price for investment goods rises, ultimately to equal the marginal utility of consumption if all output is consumed. At this point the system switches into its interior mode with output divided between consumption and investment. Thereafter the system may continue to follow a path with rising price for investment and gradually slowing rate of capital decumulation. (Of course, nothing we have said so far guarantees that only one

\footnotetext{
The point $(0, \infty)$ is also a possible equilibrium state for the system. For $K=0, p<\infty$, one has $\dot{p}=-\infty$, and the nonnegativity constraint $p>0$ becomes binding, at which point the differential equation for $p$ fails to hold. We, therefore, assume throughout the following that $K>0$
} 
such switch point will occur; this question has been studied in detail by Arrow [1].)

The time required to traverse the distance between fixed initial and terminal values $K_{0}, K_{T}$ can be shown to increase with the vertical distance of the trajectory from the fixed point $\left(K^{*}, p^{*}\right)$, near which the velocity of the system point is very low. In other words, we see that as the plan period becomes longer, the system tends to spend an increasing proportion of the period in the vicinity of the particular value $K^{*}$. Further, one might conjecture that if the problem remains meaningful even as the plan period becomes infinitely long, then the economy would tend asymptotically to this stationary point $\left(K^{*}, p^{*}\right)$. This conjecture has been verified by various authors (e.g., Cass [5], [6] and Samuelson [14]) for models of the sort we have specified in the preceding.

This argument thus yields a fairly complete solution to the question of the optimal saving policy in this simple example. The technique of analysis illustrated here is typical of the procedures in standard analyses of optimal saving policy, such as those to which we have referred before.

- Given the foregoing analysis, the task of a central planning agency with direct control over the economic system can be reduced to the following: 1) to select a value for $p_{T}$ which ensures adequate terminal capital; 2) to solve the two-point boundary value problem for the assigned value of $\left.p_{T} ; 3\right)$ to determine the associated policy $I(t)$ or $s(t)$ satisfying all the preceding necessary conditions; and 4) to dictate production plans and the composition of output in accord with that optimal policy. The agency could also, of course, attempt to synthesize the resulting open-loop control to obtain a feedback control law $I(K)$ displaying investment as a function of the capital stock at each instant. This saving policy could then be implemented by central control determining the composition of output at each instant. In the next section we consider an alternative to this procedure.

\section{A Market Mechanism}

We now wish to show that existing market institutions may realize an optimal path in the preceding problem without appeal to an agency having any direct control over resource allocation. Further, we shall observe that the system resulting from such a market mechanism corresponds to a partially synthesized control. From this there follow certain interesting questions as to the stability of the market-guided accumulation process.

Let us, therefore, imagine that the planning agency may participate in various market operations in an attempt to influence the development of the economy. In particular, we assume that whenever the flow of consumption goods is at rate $C$, the agency stands ready to buy consumption goods at a quoted price $U^{\prime}(C)$. Suppose that actual production is carried out by numerous small independent firms who take the agency's posted price for consumption goods as fixed.

Assume further that the agency stands ready to accept deposits of the consumption good, storing these for later repayment with interest at a rate equal to $\gamma$. Assume finally that at each time $t$ there exists a prevailing price $p$ for a unit of capital equipment. Our first task is to discuss the determination of this price.

Economic theory teaches us that in a system of perfect capital markets the price of such a unit of capital equipment ought to reflect the present value of all future rentals earned by the unit and ought, therefore, to be given by an expression

$$
\begin{aligned}
p(t)=\int_{t}^{T} R(\tau) \exp [-(\gamma+\delta)(\tau-t)] d \tau & \\
& +p_{T} \exp [-(\gamma-\delta)(T-t)]
\end{aligned}
$$

where $R(\tau)$ is the value of the incremental output produced by the marginal machine at time $\tau$, and $p_{T}$ is the unknown scrap value of the asset at the terminal time $T$. (The exponential decay factor reflects both time discount and physical deterioration.) Again, on economic grounds, given the prices $U^{\prime}(C)$ for consumption and $p$ for investment goods, it may be argued that the imputed rental $R(\tau)$ can be estimated by

$$
R(\tau)=\left[U^{\prime}(C) \frac{C}{F}+p \frac{I}{F}\right] F^{\prime}(K(\tau))
$$

where the bracketed term can be recognized as simply a weighted average price. Differentiating the expression for $p(t)$ with respect to $t$ and substituting for $R(t)$, we find the following:

$$
\dot{p}(t)=-\left[U^{\prime}(C) \frac{C}{F}+p \frac{I}{F}\right] F^{\prime}(K(t))+(\gamma+\delta) p
$$

with terminal condition $p(T)=p_{T}$.

Now what is the situation? The price equation given in the foregoing (excluding the terminal condition) is realized as a consequence of the operation of perfect capital markets, that is, as a result of trading by portfolio managers seeking the best investment options. The planning agency need play no role in these individual portfolio decisions and asset transfers. Further, the pursuit of maximum profit by independent producers will lead in principle (given the price quoted by the planning agency for consumption goods) to that composition of output at each instant which satisfies $U^{\prime}(C(t))=p(t)$. (If the equality cannot be realized, producers are led naturally to select the appropriate boundary case.) In other words, the pricing actions of the planning agency and the capital markets induce independent firms to act so as to maximize the Hamiltonian $H$, while the action of the agency in receiving deposits of the consumption good serves to peg the rate of return to assets in such a way as to force the asset price $p$ determined on capital markets to agree with the auxiliary variable derived earlier (at least up to boundary conditions). If, further, the planning agency announces a terminal price $p_{T}$ at which it is prepared to acquire capital goods, the agreement of the perfect asset price $p$ with the auxiliary variable $p$ is complete. Thus, by posting a price for consumption goods at each instant, a terminal price for capital goods, and by accepting deposits 
at a prescribed rate of interest, the planning agency apparently can lead a free capital market into generating a price equal to the auxiliary variable $p$, which can then in turn act as a signal to independent producers such that they are led to produce the optimal outputs of investment and consumption goods.

We thus have the makings of a rather interesting theorem, a dynamic version of a well-known result in static economics, namely, that an optimal policy for the problem posed initially can be attained by a competitive system (provided only that the planning agency develops the appropriate signals with respect to the consumption good, terminal asset values, and the appropriate rate of return on assets or rate of interest).

The introduction of additional sectors-or, for that matter, additional nonproduced nontradable assets-would not add anything to this analysis except some further conditions necessary for maximization of the Hamiltonian $H$. But the introduction of distinct durable and tradable capital goods, on the other hand, would introduce problems not encountered in our one-sector model. We must still decide upon the extent of saving and investment, as before, but now we must also decide upon the composition of investment as between the different capital goods.

Roughly speaking, when there are many different capital goods, the resolution of the optimal accumulation problem may be described as follows.

1) Specification of an optimal accumulation problem itself entails an implicit social rate of time discount implied by the criterion function, and optimal decisions may be expected to make the own-rate of return on the consumption good equal to this implied social rate of time discount, just as in the foregoing.

2) Given the own-rate of return on the consumption good pegged at the social rate of time preference, capital market trading realizes the dynamic efficiency conditions of Samuelson [15]-the arbitrage conditions or auxiliary equations - so as to force all capital goods prices to change at a rate which makes the yield on each capital good equal to that on the consumption good. This determines the prices of all capital goods, assuming that initial values can be specified in accord with terminal requirements.

3) Given the prices of all capital goods and the opportunity cost of diverting resources from consumption as indicated by the criterion function, the extent and composition of investment are determined by the actions of producers in each of the productive sectors who act so as to maximize current profit.

4) Given the outputs of each sector, the accumulation of various assets is determined by accumulation equations similar to (2), so asset stocks are all determined once initial conditions are given. Hence we have prices and endowment levels determined at each instant and the whole evolution of an optimal path established from initial conditions.

This little scenario thus sketches a way in which optimal control of a system with distinct capital goods could be realized. What we are arguing is that the requirements for an optimal path can be summarized as the following:

1) there must exist continuous asset prices such that the yield on all assets is equal, and their common value is the social rate of time discount implied by the criterion function;

2) the initial prices must be chosen to satisfy some transversality condition; and

3) with asset prices and the endowment levels given at each instant, the composition of output must be selected so as to yield a maximum value to net national product.

We are now suggesting that a decentralized system may be expected to realize condition 3) through the actions of suppliers and to equalize asset yields through arbitrage. What remains for the planning agency is simply to assign initial asset prices so as to satisfy terminal or transversality conditions and to equate the common yield on assets with the social rate of time preference or otherwise establish an appropriate price for the consumption good.

If proper representation of saving decisions and the market for funds were to bring asset yields into balance with time preference-as is theoretically the role of markets for funds, then only the assignment of initial asset prices would remain.

If, finally, future markets were able to look into the distant future and recognize the satisfaction of transversality conditions, or, more precisely, were able to recognize the eventual violation of one of the auxiliary equations as a result of failure to satisfy transversality conditions, then the assignment of initial prices would also be realized as required for optimal growth. (This issue is investigated most thoroughly by Shell and Stiglitz [18].)

In a complete system of perfect markets, therefore, central economic control becomes unnecessary; every one of the conditions for optimal growth can be realized by a decentralized system of independent decision makers. Academician L. S. Pontryagin and his colleagues have thus enunciated a newer and more powerful principle of an invisible hand; the maximum principle of Pontryagin is seen to be the culmination of a logical sequence originating in the maximum principle of Adam Smith.

Of course, it goes without saying that the principle holds only for an ideal system of perfect markets; it applies equally to a decentralized socialist economy and a free market capitalist economy. It is a theorem about allocation mechanisms and not about income distribution. But is it not fascinating that the evolution of a free enterprise system has included development of some form (often quite imperfect) of each of the market structures required in principle to realize the theoretically optimal decentralized control system? (Moreover, it is also clear that these remarks apply only to a community in which the value of output (gross national product) is the single measure of economic performance. One of the fascinating questions in economics (and in social theory) at the present time is the 
question to what extent any similar procedure of decentralization through a hierarchical system with independent decisions is feasible when the measure of economic performance has several facets reflecting environmental concerns, income and wealth distribution, equity, security, and the like. We cannot pursue this issue here.)

The literature on the general analysis stated in the preceding is relatively old. In a remarkable anticipation of the maximum principle, Samuelson and Solow [17] suggest many of the aforementioned ideas in their analysis of the Ramsey problem with heterogeneous capital goods. The dynamic efficiency conditions wére also developed in a model [15] with no explicit criterion functional depending upon consumption but where the objective is the weaker criterion of efficiency familiar from the literature on "turnpike" theorems. (See, e.g., Burmeister and Dobell [3, ch. 10].)

Radner [13] and Chakravarty [7] deal with the issue of optimal growth in multicapital models, and a great deal of further analysis is contained in [20].

A great many interesting issues flow from these considerations; we can sketch only a few here. Perhaps the essential point is that this decentralized system without central control is described by a different set of equations than the centralized system under optimal control. With fully synthesized control the operations of our model economic system can be described by

$$
\begin{aligned}
\dot{K} & =s(K) F(K)-\delta K \\
K(0) & =K_{0} \\
C & =F(K)-\dot{K}-\delta K
\end{aligned}
$$

where $s(K)$ is the optimal control obtained by integrating out the auxiliary variables and expressing the control as a function of the state alone. (The initial condition $K_{0}$ is imposed by nature.) Under the decentralized scheme outlined in the preceding, however, the operation of the system must be described by the equations

$$
\begin{aligned}
\dot{K} & =s(p, K) F(K)-\delta K \\
K(0) & =K_{0} \\
C & =F(K)-\dot{K}-\delta K \\
\dot{p} & =-U^{\prime}([1-s(p, K)] \cdot F(K)) F^{\prime}(K)+(\gamma+\delta) p \\
p(T) & =p_{T}
\end{aligned}
$$

where $s(p, K)$ is obtained from the condition

$$
U^{\prime}((1-s) F(K))=p .
$$

In other words, the decentralized system corresponds to one with incompletely synthesized control in which the auxiliary variables appear explicitly. Because the original and auxiliary equations generally form a stable-unstable pair (a system with a saddle point equilibrium), and because the terminal condition on the auxiliary variable is not imposed by nature, a number of stability questions arise. We deal with these and other issues in the next section.

\section{FURTHER ISSUES}

\section{A. Stability of the System Under Decentralized Control}

We have suggested that the market mechanisms present in an idealized capitalist system may realize in a decentralized fashion some or all of the control necessary to guide the system along an optimal trajectory. With many heterogeneous capital goods, the common own-rate established by asset trading must be brought into balance with the social rate of time preference along an optimal path, and, in addition, initial prices must be assigned in conformity with terminal conditions. These latter two conditions arc not naturally satisfied by a descriptive model of a market system. Whether a capitalist system has in principle a mechanism to meet these conditions and the consequences if it has not have been subjects for recent discussion.

Hahn [9] showed that in a model where no explicit intertemporal optimization is considered at all but where an arbitrary hypothesis determining total saving instead serves to determine the common own-rate of return at each instant and where transversality conditions are not considered but initial asset prices are instead taken to be historically given, then the system does not, in general, converge to a stable equilibrium, a so-called "balanced growth path." Rather, it diverges from the "balanced growth" equilibrium which is a saddle point in the price-quantity space. Hahn concluded that the presence of many capital goods was responsible for this result and apparently felt that the result itself cast doubt upon the desirability of a system of capitalist markets for guiding a complex economy along an accumulation path.

Shell and Stiglitz [18] argued that the result was a consequence of incomplete specification, that full specification of an idealized capitalist system should include possible futures markets, and that if equilibrium in all of a complete set of futures markets were required, then only the unique initial prices leading to balanced growth would be tenable as an initial price assignment in a competitive capitalist system.

Burmeister et al. [4] suggested that the result of Hahn should be assessed in terms of its specification as to portfolio behavior, which requires asset holders to be prepared to accept the promise of capital gains (confidently expected) at an infinite rate as a perfect offset to an infinite gap between actual earning streams of two different assets. They showed that an alternative (still unrealistic) assumption concerning the determination of investment composition results in convergence of the system to a globally stable balanced growth path.

Still another very recent approach is based on $[3$, sec. 9-5, pp. 297-306]. It has been proved that the manifold consisting of the backward solutions from stationary equilibrium is generally of higher dimension if all capital gains are not saved. Accordingly, systems in which consumption is sensitive to capital gains income may be "more stable," a result that is not intuitively obvious. The interested reader is referred to Burmeister et al. [21]. 
We do not propose to pursue these issues here, save to observe that the results turn on the question of whether one is dealing with a completely descriptive system taking initial asset prices as given, or with a planning model having some mechanism for selecting initial values for asset prices in conformity with terminal conditions. Obviously, the question of stability arises in the planning problem with central control only as a computational difficulty; in a descriptive system or under decentralized control without continuing recomputation of all prices, however, instability may be a crucial feature of the accumulation process.

\section{B. Joint Production and Nonuniqueness}

Recently it has been shown (Kurz [11], Gale and Sutherland $[8])$ that in some cases a stationary solution $\left(K^{*}, p^{*}\right)$ to the Euler-Lagrange equations need not be unique, a circumstance which raises doubts as to the significance of so-called turnpike theorems asserting characteristic features of optimal paths. However, these results have generally been derived in models having a criterion (welfare) function in which not only consumption flows but also state variables such as wealth or capital stocks enter. It may be argued that such models are objectionable on economic grounds in that they "double-count" the benefits of additional stockspresumably increments to wealth or capital stock at a point in time increase welfare only to the extent that they contribute to future consumption, the value of which is already measured in the criterion functional $J$.

Rather than pursue this issue directly, we shall retain our previous criterion functional and show that the same nonuniqueness property may result under slightly different technological assumptions. More precisely, (1) of our earlier model may be replaced by a production possibility frontier in the form

$$
C=G(I, K) \text {. }
$$

The function has a simple interpretation: at any fixed time $t, K$ is fixed; given a nonnegative (admissible) value of gross investment $I$, the value of $G$ gives the maximum technologically attainable consumption flow $C$. Equation (1) then is seen to be written in a form which implicitly imposes the assumptions that a reduction in the flow $I$ by one unit results in a corresponding increase in the flow $G$ by exactly one unit. This strong assumption may be replaced by the weaker hypothesis that the flows $C$ and $I$ are outputs produced jointly by a single production process, in which case economists generally presume that $G$ is a strictly concave function in $I$ and $K$. Samuelson [16] has proved that the singularity of the Hessian matrix of $G$ is a necessary and sufficient condition for the absence of joint production. ${ }^{2}$ Thus, when $G$ is strictly concave (and its

\footnotetext{
${ }^{2}$ Strictly speaking, in making this observation we are implicitly assuming the existence of an underlying fixed labor force which is fully employed so that all the variables $C, I$, and $K$ mav be interpreted for our purposes as per-capita quantities; a production possibility frontier defined with a variable labor force appearing explicitly as an argument of the function $G$ homogeneous of degree one in all its arguments is not strictly concave.
}

Hessian matrix is everywhere negative definite), joint production of the sort described does exist.

Now consider the Euler-Lagrange equations for a problem of the sort analyzed earlier, assuming for simplicity that the constant $\delta$ is zero. At a stationary point, where $\dot{K}=\ddot{K}=0$, we must have

$$
\gamma G_{I}\left(0, K^{\prime}\right)+G_{K^{\prime}}\left(0, K^{\prime}\right)=0
$$

which yields the condition

$$
\Phi_{K}=0
$$

and (18) need not have a unique positive solution if $\Phi_{K}$ changes sign. But by direct calculation,

$$
\Phi_{K}=\gamma G_{I K}+G_{K \kappa}
$$

and thus if $\gamma>0, \Phi_{K}$ may indeed change sign. (When $\gamma=0$, of course, the restriction $G_{K K}<0$ implies $\Phi_{K}<0$ everywhere.)

It is interesting, however, that such a possibility exists only if there is joint production and $G$ is a strictly concave function. To prove this assertion, we define the net own-rate of return on capital by $r=G_{K} / G_{I}$ and note that (17) holds if and only if $r=\gamma$. But since it is known that $d K / d r \mid \dot{K}=0$ $<0$ in models without joint production, the proof is immediate; we conclude (18) has a unique solution $K^{*}$ under these circumstances. (See [2] and [3, ch. 9].)

Where joint production does exist, however, the possibility of two (or more) roots $K^{*}$ and $K^{* *}$, both satisfying (17) and (18), is definitely present. The existence of multiple stationary solutions to the Euler-Lagrange equations also suggests that the optimal policy may be altered drastically by the initial condition $K_{0}$, with the optimal $K(t)$ path arching toward a lower root $K^{*}$ when $K_{0}$ is sufficiently low but arching toward a higher root $K^{* *}$ if $K_{0}$ is sufficiently high. (A more detailed discussion of this issue is given in [3, pp. 411-416], and a formal treatment can be found in [22].)

Further investigation of this subject may thus yield some insights into the adequacy of conclusions as to development policies and the efficiency of foreign aid derived from simple aggregate models of the process of economic development. Numerical examples illustrating the extreme sensitivity of model results to parameter value changes when the preceding conditions of nonuniqueness prevail also lead to question whether such results are representative of actual development problems.

\section{Robust Controls}

Finally, we shall close this section with the observation that the search for optimal policies becomes more difficult as more complex portfolio decision, investment decision, or saving decision rules are introduced and still more so as one attempts to take account of uncertainty. Eventually the search will turn from a concern with exact optimality to a concern with the identification of policies which lead to satisfactory values of the criterion function and which are robust against shocks or possible mispecification. This search can be expected to bring economists closer to the 
work of control engineers seeking policies which, while not precisely optimal, may have the property of inexorably reducing some convenient measure of distance from a target. and to work of numerical analysts seeking "methods" which yield "good" results for a whole class of problems, though not necessarily optimal results for any one problem in that class, or to work on the concept of fuzzy sets and algorithms, which may permit approximate solution of approximately specified problems.

\section{Concluding Comments}

A developed economy with elaborate goods and capital markets represents a very complex structure indeed. Within the market framework individual producers and suppliers of goods or services contend with individual households allocating expenditures. Each agent represents a conscious decision process guided by more or less well-defined goals. From these myriad individual decisions there emerges an explicit allocation of resources and composition of national output. Under the thrust of these allocation and investment consequences, in turn, the whole economy grows and evolves.

In the present limited space, and in a very impressionistic fashion, we have tried to suggest the flavor of a number of issues in control theory that arise from such framework. We have sketched a model which might crudely describe the process of capital accumulation in a highly aggregated economy and have reviewed the solution to a problem in optimal control of such a model. We then noted that market institutions working through the decisions of individuals might permit a central planning agency to rely on only very generalized tools of economic policyinstruments like money supplies and tax rates - even while accomplishing optimal control of the system. Fascinating issues of controllability can arise in these circumstances, questions whether individuals in the system may adjust their own decisions so as to cushion the effect of such control as is exercised and render the overall system yet more difficult to guide.

Decentralized systems based on shadow pricing algorithms were seen to have their counterpart in market institutions; in both interpretations questions of stability and of the existence of adequately robust controls arise.

The basic point, however, is that a market mechanism is seen to eliminate to some extent the need for central controlling authority even for the attainment of strictly technocratic goals. The principle of the invisible hand carries as a corollary the principle that coercive power over individual decisions is unnecessary for economic purposes.

We must not overstate the case, however. We have not sketched a mechanism which does away altogether with a central planning agency; some procedure for computing initial prices and assuring appropriate total saving rates in the community remains essential to our theoretical analysis. Nor could it really be argued that the power to tax and transfer income is consistent with totally free economic decisions; growth policies and saving or investment targets attained through use of such powers can, therefore, not be considered to be fully decentralized.

Nevertheless, we find it somewhat remarkable that market institutions evolving naturally through centuries of social development mirror as faithfully as they do the conditions for optimality in complex systems. Decomposition algorithms, dual programs, and auxiliary variables were in everyday use in solving large-scale constrained maximization problems long before their theoretical foundations were erected. It seems safe to predict that economic systems will prove to be a continuing source of fascinating problems for the sophisticated control theorist. We hope these rough notes serve to identify a few questions of this sort.

\section{REFERENCES}

[1] K. J. Arrow, "Applications of control theory to economic growth," in Mathematics of the Decision Sciences, vol. 12, G. Dantzig and A. Veinott, Eds. Providence, R.I.: American Mathematical Society, 1968, pp. 85-119.

[2] E. Burmeister, "The existence of golden ages and stability in the two-sector model," Quart. J. Econ., vol. 81, Feb. 1967, pp. 146154.

[3] E. Burmeister and A. R. Dobell, Mathematical Theories of Economic Growth. New York: Macmillan, 1970.

[4] E. Burmeister, A. R. Dobell, and K. Kuga, "A note on the global stability of a simple growth model with many capital goods," Quart. J. Econ., vol. 82, Nov. 1968, pp. 657-665.

[5] D. Cass, "Optimum growth in an aggregative model of capital accumulation," Rev. Econ. Stud., vol. 32, July 1965, pp. 233-240.

[6] - "Optimum growth in an aggregative model of capital accumulation: a turnpike theorem," Econometrica, vol. 34 , Oct. 1966, pp. 833-850.

[7] S. Chakravarty, "Optimal programme of capital accumulation in a multisector economy," Econometrica, vol. 33, Sept. 1962, pp. $338-355$.

[8] D. Gale and W. R. Sutherland, "Analysis of a one-good model of economic development," in Mathematics of the Decision Sciences, vol. 12, G. Dantzig and A. Veinott, Eds. Providence, R.I. American Mathematical Society, 1968.

[9] F. H. Hahn, "Equilibrium dynamics with heterogeneous capital goods," Quart. J. Econ., vol. 80, Nov. 1966, pp. 633-646.

[10] F. H. Hahn and R. C. O. Matthews, "The theory of economic growth: a survey," Econ. J., vol. 74, Dec. 1964, pp. 779-902.

[11] M. Kurz, "Optimal economic growth and wealth effects," Int. Econ. Rev, vol, 9. Oct. 1968, pp, 348-357.

[12] O. L. Mangasarian, "Sufficient conditions for the optimal control of nonlinear systems," J. Soc. Ind. Appl. Math. (Contr.), vol. 4, 1966, no. 1, pp. 139-152

[13] R. Radner, "Optimal growth in a linear-logarithmic economy," Int. Econ. Ret., vol. 7, Jan. 1966, pp. 1-33.

[14] P. A. Samuelson, "A caternary turnpike theorem involving consumption and the golden rulc," Amer. Econ. Rev, vol 55, June 1965, pp. 486-496.

[15] - "Efficient paths of capital accumulation in terms of the calculus of variations," in Mathematical Methods in the Social Sciences, K. Arrow, S. Karlin, and P. Suppes, Eds. Stanford, Calif.: Stanford Univ. Press, 1960, pp. 77-88.

[16] - "The fundamental singularity theorem for non-joint production," Int. Econ. Rev., vol. 7, Jan. 1966, pp. 34-41.

[17] P. A. Samuelson and R. Solow, "A complete capital model involving heterogeneous capital goods," Quart. J. Econ., vol. 70, Nov. 1956 , pp. $537-562$.

[18] K. Shell and J. E. Stiglitz, "The allocation of investment in a dynamic economy," Quart. J. Econ., vol. 71, Nov. 1967, pp. 592-609.

[19] R. M. Solow, "A contribution to the theory of economic growth," Quart. J. Econ., vol. 70, Feb. 1956, pp. 65-94.

[20] "Symposium on optimal infinite programmes," Rer. Econ. Stud Jan. 1967; also F. H Hahn and F. P. Brechling, Eds., Problems in the Theory of Optimal Accumulation. London: Oliver and Boyd, 1967.

[21] E. Burmeister, C. Caton, A. R. Dobell, and S. Ross, "The "saddle point property' and the structure of dynamic heterogeneous capital good models," Econometrica, to be published.

[22] N. Liviatan and P. A. Samucison, "Notes on turnpikes: stable and unstable," J. Econ. Theory, vol. 1, Dec. 1969, pp. 454-476. 